\title{
THE BITANGENTS AT A POINT OF A DESMIC SURFACE
}

BY R. M. MATHEWS

A plane tangent to a surface of the fourth order cuts the surface in a curve of the fourth order with a singular point at the point of contact. Through this point there can then be drawn six lines tangent to the curve elsewhere. So through a point on a surface of the fourth order there are in general six bitangents.

The second points of contact of these bitangents lie on a conic which, for a desmic surface, degenerates into two lines. Thus we have two sets of three bitangents; numerous geometrical properties of one triple have been given by G. Humbert and others. ${ }^{*}$ Concerning the second triple only some general properties that apply to both sets are known; no characterizing geometric properties have been published. This paper presents one.

For brevity in the equations we shall use the symbol $\overline{i j}$ to denote $\left(x_{i}^{2}-x_{j}^{2}\right)$.

The pencil of desmic surfaces in variables $(y)$ and parameter $(x)$,

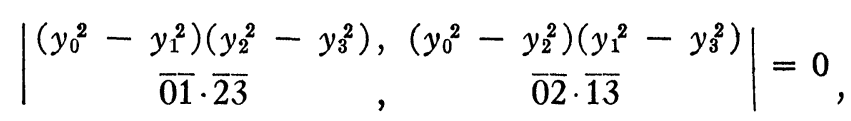

determines a cubic complex, and the cubic cone of this complex of which an arbitrary point $P(x)$ is the vertex, cuts the plane $y_{3}=0$ in the cubic curve

(2) $\overline{0} \overline{3} y_{1} y_{2}\left(x_{2} y_{1}-x_{1} y_{2}\right)+\overline{13} y_{2} y_{0}\left(x_{0} y_{2}-x_{2} y_{0}\right)$

$$
+\overline{2} 3 y_{0} y_{1}\left(x_{1} y_{0}-x_{0} y_{1}\right)=0 \text {. }
$$

* Humbert, G., Sur la surface desmique du quatrième ordre, Journal de Mathématiques, (4), vol. 7 (1891), pp. 353-398.

Jessop, C. M., Quartic Surfaces with Singular Points, Cambridge, 1916.

Mathews, R. M., Cubic curves and desmic surfaces, Transactions of this Society, vol.28 (1926), pp. 502-522, and ibid., vol. 30 (1928), pp. 19-23. 
The tangent plane at $P(x)$ to the desmic surface through $P$ is

(3) $\overline{1} \overline{3} \cdot \overline{2} \overline{3} \cdot \overline{1} \overline{2} x_{0} y_{0}+\overline{2} \overline{3} \cdot \overline{0} \overline{3} \cdot \overline{2} \overline{0} x_{1} y_{1}$

$$
+\overline{0} \overline{3} \cdot \overline{1} \cdot \overline{0} \cdot \overline{1} x_{2} y_{2}+\overline{01} \cdot \overline{1} \overline{2} \cdot \overline{20} x_{3} y_{3}=0,
$$

and cuts $y_{3}=0$ in a line $A B C$ with

(4) $\left\{\begin{array}{l}A: \overline{0} \overline{3} x_{1} x_{2}: \overline{1} \overline{3} x_{2} x_{0}: \overline{2} \overline{3} x_{0} x_{1}, \\ B: \overline{0} \overline{3}\left(x_{1} x_{2}+x_{0} x_{3}\right): \overline{1} \overline{3}\left(x_{0} x_{2}+x_{1} x_{3}\right): \overline{2} \overline{3}\left(x_{0} x_{1}+x_{2} x_{3}\right), \\ C: \overline{0} \overline{3}\left(x_{1} x_{2}-x_{0} x_{3}\right): \overline{1} \overline{3}\left(x_{0} x_{2}-x_{1} x_{3}\right): \overline{2} \overline{3}\left(x_{0} x_{1}-x_{2} x_{3}\right) .\end{array}\right.$

The points $A, B, C$ are on the cubic curve (2) and the lines $P A, P B, P C$ are the known bitangents.*

The cubic curve (2) has for Hessian curve the cubic

$$
\left|\begin{array}{l}
\overline{2} \overline{3} x_{1} y_{1}-\overline{1} \overline{3} x_{2} y_{2}, \overline{2} \overline{3}\left(x_{1} y_{0}-x_{0} y_{1}\right), \overline{1} \overline{3}\left(x_{0} y_{2}-x_{2} y_{0}\right) \\
\overline{2} \overline{3}\left(x_{1} y_{0}-x_{0} y_{1}\right), \overline{0} \overline{3} x_{2} y_{2}-\overline{2} \overline{3} x_{0} y_{0}, \overline{0} \overline{3}\left(x_{2} y_{1}-x_{1} y_{2}\right) \\
\overline{1} \overline{3}\left(x_{0} y_{2}-\dot{x}_{2} y_{0}\right), \overline{0} \overline{3}\left(x_{2} y_{1}-x_{1} y_{2}\right), \overline{1} \overline{3} x_{0} y_{0}-\overline{0} \overline{3} x_{1} y_{1}
\end{array}\right|=0 .
$$

To write the coordinates of an arbitrary point $Q$ on the line $A B C$, it is convenient to find $\bar{A}$ as the harmonic conjugate of $A$ with respect to $B$ and $C$, namely

$$
\bar{A}: \overline{0} \overline{3} x_{0} x_{3}: \overline{1} \overline{3} x_{1} x_{3}: \overline{2} \overline{3} x_{2} x_{3}
$$

and then to write the coordinates for

$$
Q \equiv \rho A+\sigma \bar{A} \text {. }
$$

On solving $A \bar{A}$ simultaneously with the Hessian (5), we find that the values of the $\rho / \sigma$ ratio for the points of intersection are roots of the cubic

(7) $2 \rho^{3} x_{0} x_{1} x_{2} x_{3}+\rho^{2} \sigma \sum x_{0}^{2} x_{1}^{2}+6 \rho \sigma^{2} x_{0} x_{1} x_{2} x_{3}$

$$
+\sigma^{3}\left(\sum x_{0}^{2} x_{1}^{2}-\sum x_{0}^{4}\right)=0 .
$$

Next, the line $P Q$ is an arbitrary line through $P$ on the tangent plane at $P$. We write the coordinates of an arbitrary point $R$ on $P Q$ in the form

$$
R \equiv \lambda Q+\mu P
$$

\footnotetext{
* For these equations and results see Mathews, loc. cit.
} 
and substitute in the equation of the desmic surface. The result is a fourth degree equation in the ratio $\lambda / \mu$ whose four roots give the intersections $R$ of $P Q$ with the surface. Since $P$ is a double point on the intersection of the plane and surface this quartic equation reduces to the quadratic

$$
\left\{\begin{array}{l}
\left.\lambda^{2}\left\{\left(z_{0}^{2}-z_{1}^{2}\right) z_{2}^{2} n-\left(z_{0}^{2}-z_{2}^{2}\right) z_{1}^{2} m\right)\right\} \\
\quad+2 \lambda \mu\left\{\left[\left(z_{0}^{2}-z_{1}^{2}\right) z_{2} x_{2}+z_{2}^{2}\left(x_{0} z_{0}-x_{1} z_{1}\right)\right] n\right. \\
\left.\quad-\left[\left(z_{0}^{2}-z_{2}^{2}\right) z_{1} x_{1}+z_{1}^{2}\left(x_{0} z_{0}-x_{2} z_{2}\right) m\right]\right\} \\
\quad+\mu^{2}\left\{\left[\left(z_{0}^{2}-z_{1}^{2}\right) \overline{23}+z_{2}^{2} \overline{0} \overline{1}+4\left(x_{0} z_{0}-x_{1} z_{1}\right) x_{2} z_{2}\right] n\right. \\
\quad-\left[\left(z_{0}^{2}-z_{2}^{2}\right) \overline{13}+z_{1}^{2} \overline{02}\right. \\
\left.\left.\quad+4\left(x_{0} z_{0}-x_{2} z_{2}\right) x_{1} z_{1}\right] m\right\}=0,
\end{array}\right.
$$

where $m=k \overline{0} \overline{2} \cdot \overline{1} \overline{3}, n=k \overline{0} \overline{1} \cdot \overline{2} \overline{3}$, ( $k$ a factor of proportionality), and where the $z$ 's are the coordinates of $Q$.

Now, when $P Q$ is a bitangent to the surface the two roots of this quadratic are equal. We set the discriminant equal to zero, substitute for the $z$ 's the coordinates of $Q$ as

$$
Q=\rho A+\sigma \bar{A}
$$

and obtain an equation of the sixth degree in the ratio $\rho / \sigma$. Three of the roots are $\sigma=0$ and $\rho / \sigma= \pm 1$ giving $A, B, C$, the points for the known bitangents, and leaving for the determination of the other three exactly equation (7) which is the condition that $Q$ lie at the intersections of the line $A B C$ with the Hessian cubic.

The cubic cone at $P$ cuts an arbitrary plane, not through $P$, in a cubic curve which has a definite Hessian; when the points of the latter are joined to $P$ we get a cubic cone which may be called the Hessian cone corresponding to the first. Thus we have the following theorem.

THeOREM. The bitangents to a desmic surface at a point thereon lie in two triples; the lines of one triple lie on the cubic cone of the system proper to $P$, and of the other on the corresponding Hessian cone.

WEST VIRGINIA UNIVERSITY 